\title{
PET Studies of Parkinsonian Patients Treated with Autologous Adrenal Implants
}

\author{
M. Guttman, R.S. Burns, W.R.W. Martin, R.F. Peppard, M.J. Adam, T.J. Ruth, \\ G. Allen, R.A. Parker, N.B. Tulipan and D.B. Calne
}

\begin{abstract}
Transplantation of autologous adrenal medulla tissue into the striatum has recently been proposed as a treatment for Parkinson's disease. We report the use of positron emission tomography (PET) to evaluate patients who had adrenal implants placed into the right caudate. 6-[18F] fluoro-L-dopa (6-FD) scans were performed to study the integrity and activity of the implant, and the nigrostriatal dopamine system before and six weeks after transplantation surgery. [ ${ }^{68} \mathrm{Ga}$ ] Gallium-ethylenediaminetetraacetate $(\mathrm{Ga})$ scans were also performed to assess the blood brain barrier. The Ga scans performed on two patients showed increased permeability of the blood brain barrier at the surgical site. 6-FD PET scans in five patients did not show a consistent change in striatal uptake following adrenal medullary implantation after six weeks. Further assessment of implant viability with 6-FD PET scans after longer follow up may provide useful information if the blood-brain barrier becomes re-established with the passage of time.
\end{abstract}

RÉSUMÉ: Étude par PET scan de patients parkinsoniens traités par implant autologue de tissu surrénalien La transplantation de tissu surrénalien autologue dans le striatum a été proposée récemment comme traitement de la maladie de Parkinson. Nous avons utilisé la tomographie par émission de positrons (PET scan) pour évaluer les patients qui ont reçu des implants surrénaliens dans le noyau caudé droit. Des scans à la 6-| 18 F \}fluoro-L-dopa (6-FD) ont été effectués pour étudier l'intégrité et l'activité de l'implant et du système dopaminergique nigrostrié avant et six semaines après la chirurgie. Des scans à la $\left\{{ }^{68} \mathrm{Ga}\right\}$ éthylènediaminetétra-acétate de Gallium (Ga) ont également été effectués pour évaluer l'intégrité de la barrière hémo-encéphalique. Des scans au Ga pratiqués chez deux patients ont montré une perméabilité accrue de la barrière hémo-encéphalique au niveau du site de la chirurgie. Des scans au 6-FD faits chez 5 patients n'ont pas mis en évidence de changement univoque de la captation au niveau du striatum six semaines après l'implantation de cellules de la médullo-surrénale. Des évaluations ultérieures de la viabilité de l'implant au moyen de scans à la 6-FD au cours d'un suivi à long terme pourront fournir des informations utiles si la barrière hémo-encéphalique se rétablit avec le temps.

Can. J. Neurol. Sci. 1989; 16: 305-309

Neuronal grafting has been reported to ameliorate the symptoms of parkinsonism in experimental subjects.' Since the initial report of clinical improvement after autologous adrenal implantation into the brain of Parkinsonian patients, ${ }^{2}$ there has been intense interest in the use of this surgical therapy for Parkinson's disease. The early enthusiastic outlook has been somewhat subdued in the light of subsequent reports of variable success. $3,4,5,6,7,8$ There have been high mortality and morbidity rates at some centres. In order to evaluate the efficacy of autologous adrenal implantation, it is important to have accurate methods of assessing graft function post-operatively. Many investigators have utilized clinical rating scales, ${ }^{2}$ neurophysiological testing, ${ }^{8}$ cerebrospinal fluid measurements of catecholes and their metabolites from lumbar ${ }^{8,9}$ and Omaya reservoir samples $7,9,10$ as well as dopamine receptor binding measurements with positron emission tomography. ${ }^{8}$ The ultimate analytic technique would assess the functional aspects of the neural implant in vivo. Since the tissues being used for the procedure are chosen for their ability to produce dopamine, it should be rewarding to monitor dopamine metabolism.
Positron emission tomography (PET) allows the study of the regional concentration of radioisotopes in the living brain. 6[18F] fluoro-L-dopa (6-FD) has been used to evaluate the integrity of the nigrostriatal dopamine pathway." Differences have been found in the striatal uptake of this compound between subjects with parkinsonism and controls. $12.13,14 \mathrm{We}$ have employed PET in the assessment of patients before and after autologous adrenal implantation for Parkinson's disease. In addition to evaluating the nigrostriatal dopamine system with 6 FD, we have utilized [ ${ }^{68} \mathrm{Ga}$ ] gallium-ethylenediaminetetraacetate (EDTA) $(\mathrm{Ga})$ to assess the integrity of the blood brain barrier. Using these in vivo techniques we have identified difficulties in the interpretation of 6-FD PET studies after surgery due to alterations of the blood brain barrier.

\section{METHODS}

\section{Patient Population}

Five patients were studied at the UBC/TRIUMF PET Centre before and after autologous adrenal implantation. All patients

From the UBC/TRIUMF PET Group, and the Division of Neurology, Department of Medicine, University of British Columbia, Vancouver (M.G., W.R.W.M., R.F.P., M.J.A., T.J.R., D.B.C.) and the Department of Neurosurgery and Neurology, Vanderbilt University, Nashville, Tennessee (R.S.B., G.A., R.A.P., N.B.T.)

Received September 2, 1988. Accepted in final form May 7, 1989

Reprint requests to: M. Guttman, 3801 University, Montreal, Quebec, Canada H3A 2B4 
were part of the Vanderbilt University Transplant Project. The patient characteristics are listed in Table 1. After the initial PET study in Vancouver, the patients returned to Nashville for their surgery. All patients had their right adrenal gland removed and had implantation of adrenal medulla tissue into the right caudate nucleus. An Omaya reservoir was placed into the left ventricle in order to sample cerebrospinal fluid post-operatively. Six weeks after surgery the patients returned for repeat PET studies.

\section{Patient Evaluation}

The initial post-operative clinical characteristics of these patients have been reported. ${ }^{7}$ The clinical signs of parkinsonism were assessed using a modified Columbia Rating Scale. ${ }^{15}$ Patients were examined before and at 3 month intervals after surgery. In general, there were 3 examinations on alternate days during a single week for each patient. In the case of patient 2 , only one or two assessments were available. Patients were examined in the morning prior to their first dose of L-dopa for that day. The one patient with on-off reaction, patient 1 , was assessed in the off-state. The median total rating scale score at baseline, 3 months and 12 months is presented in Table 1. A formal 6 week score was not performed by the same observers and is therefore not comparable to the other values.

Based on patient variability for visits close together in time, it was estimated that a change of 10 points in the total Columbia Rating Scale score was unlikely to occur by chance and indicated a definite change. A reduction in the total score represents improvement and an increase represents deterioration.

\section{PET and MRI Studies}

The PET protocol included a 6-FD study pre-operatively, followed by a scan six weeks after surgery with the same compound. Two patients had, in addition, Ga scans at the same time as their post-operative 6-FD study. The PET scans were performed on the UBC/TRIUMF PETT VI tomograph ${ }^{16}$ using a previously published protocol. ${ }^{13}$ 6-FD was prepared via the fluorination of a mercury-dopa derivative with 18F-acetyl hypofluorite. ${ }^{17} \mathrm{Ga}$ was prepared from an on-site generator purchased from New England Nuclear-Dupont Limited. For each dose, the germanium gallium generator was eluted with 0.005 molar Sodium-EDTA solution (pH 7.04).

After informed consent, patients initially had magnetic resonance imaging (MRI) performed with a Picker 0.15 Tesla cryogenic magnet. The patients were oriented in the MRI headholder so that the orbital-meatal line was parallel to the plane of the magnet. Inversion recovery sequences enabled accurate anatomical localization of the body of the putamen and external landmarks were applied to correspond with this location.

The 6-FD scans were performed with carbidopa pre-treatment (100 mg orally) one hour before injection of the isotope to prevent peripheral decarboxylation of the compound. Antiparkinsonian medication was withheid from the night before. Patients were positioned in the tomograph using the external markings determined from the MRI study. This technique enabled sampling of the same anatomical structures in all subjects. The head was fixed in place with the use of a thermoplastic mask. An intravenous catheter was placed in the opposite arm for injection of the radioisotope. Post-operatively, patients, had the identical protocol of MRI and PET scans using the same positioning techniques.

Two patients had Ga scans as part of their post-operative assessment. For these studies, the patients were positioned in the same way as the 6-FD studies. Ga was injected intravenously $(4-5 \mathrm{mCi})$ and ten scans of ten minutes duration were performed with alternating chair positions so that the anatomical levels would be comparable to the 6-FD studies. Summed scans were used for the identification of abnormalities of the blood brain barrier.

\section{PET Analysis}

Elliptical regions of interest $\left(1.2 \mathrm{~cm}^{2}\right)$ were placed on the striatum and occipito-parietal cortex on each of the four slices containing striatal activity that commenced at 110 minutes and 120 minutes after 6-FD injection. This permitted evaluation of the data from the brain regions acquired from each of the two chair positions. The average striatal radioactivity in excess of background was estimated by subtracting the activity in the background regions of occipito-parietal cerebral cortex from the striatal region. This value was then normalized to the occipitoparietal cortex value. In the background regions, because of the lack of dopa-decarboxylase activity, the only radioactive components are thought to be 6-FD and its metabolite 3-O-methylfluorodopa (3-OMFD). ${ }^{18}$ With an intact blood brain barrier, the striatal concentrations of 6-FD and 3-OMFD are thought to be the same as the occipito-parietal cortex concentrations because the transport system is similar. ${ }^{19}$ By subtracting the occipitoparietal value from the striatal value, one is left with the striatal dopamine-related metabolites of 6-FD. Each striatum was evaluated for each of the two scan times and the ipsilateral values. were then averaged to obtain a left and right striatal measurement. This strategy estimates the functional integrity of the dopamine-synthesizing cells. The same methods were applied to the pre- and post-operative scans. The occipito-parietal cortex was chosen for the reference region rather than the cerebellum because of the previous observations. ${ }^{20}$ The activity in the cerebellum has different uptake characteristics compared to areas of cerebral cortex and striatum after the administration of 6-FD, permitting potential errors using the analytic techniques outlined above.

Table 1: Clinical Characteristics Before Transplantation

\begin{tabular}{|c|c|c|c|c|c|c|c|c|}
\hline \multirow[t]{2}{*}{ Patient } & \multirow[t]{2}{*}{ Age } & \multirow[t]{2}{*}{ Sex } & \multirow{2}{*}{$\begin{array}{r}\text { H \& Y } \\
\text { Stage }\end{array}$} & \multicolumn{5}{|c|}{ Modified Columbia Scores } \\
\hline & & & & Time 0 & 3 Mo & $6 \mathrm{Mo}$ & 9 Mo & $12 \mathrm{Mo}$ \\
\hline 2. & 42 & $\mathbf{M}$ & 1 & 18 & 4 & $\mathrm{~N} / \mathrm{E}$ & $\mathrm{N} / \mathrm{E}$ & 3 \\
\hline 3. & 35 & $M$ & 2 & 28 & 1 & -1 & -7 & -4 \\
\hline 5. & 46 & $\mathrm{~F}$ & 4 & 55 & -22 & -22 & -13 & -14 \\
\hline
\end{tabular}

After the baseline examination the Modified Columbia Scores are expressed as the amount of change time 0 . 


\section{RESULTS}

\section{Patient Characteristics}

One out of the five patients, patient 5 , showed improvement at 3 months and at 12 months. The other patients were unchanged. None met the criterion for clinical deterioration. In patient 5 , improvement was seen in bradykinesia, rigidity, posture, balance and gait at 3 months and the changes were present at 12 months except for rigidity. Although the changes were apparent at 3 months after surgery, they occurred so slowly that the time of onset could not be determined precisely. By design, antiparkinsonian medications were not to be changed during the first year following the transplant, although patients 3,4 , and 5 were taken off medication for 2-14 days perioperatively. Patient 5 was receiving a total daily dose of $500 \mathrm{mg}$ of L-dopa combined with carbidopa during the baseline period and a dose of $350 \mathrm{mg}$ at 3 months and 12 months.

\section{6-FD Scans}

Examples of pre- and post-operative 6-FD scans are shown in Figure 1. Results of the analysis of the 6-FD PET studies are shown in Table 2. 6-FD PET scans six weeks after surgery showed an increase in striatal uptake in three patients but this was virtually the same on the left (non-operated) side as on the right side. Two patients showed decreased striatal uptake on the follow up study. The amount of change between the pre- and post-operative scans was not significant using the Wilcoxon Signed Rank test.

\section{Ga Scans}

Examples of the Ga scans in two patients are shown in Figure 2. Comparison of 6-FD scans with Ga scans performed in the same patient, in the same positions, is shown in Figure 3. Uptake of $\mathrm{Ga}$ was increased in the region of the transplantation site and surgical tract. When 6-FD scans are compared to these scans, there is a similar distribution of altered radioactive uptake in these regions.

\section{Discussion}

This study has employed PET scans with 6-FD to evaluate the nigrostriatal dopamine system in patients undergoing autologous adrenal implantation for Parkinson's disease. 6-FD is an analog of L-dopa and can be employed to evaluate the function of the nigrostiatal dopamine system." 6 -FD, and L-dopa cross

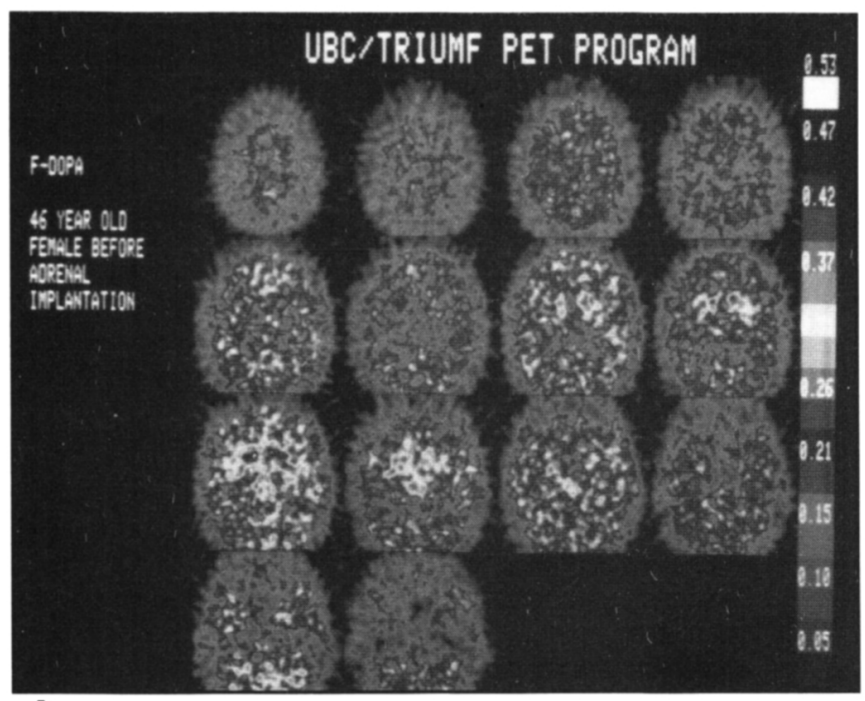

A

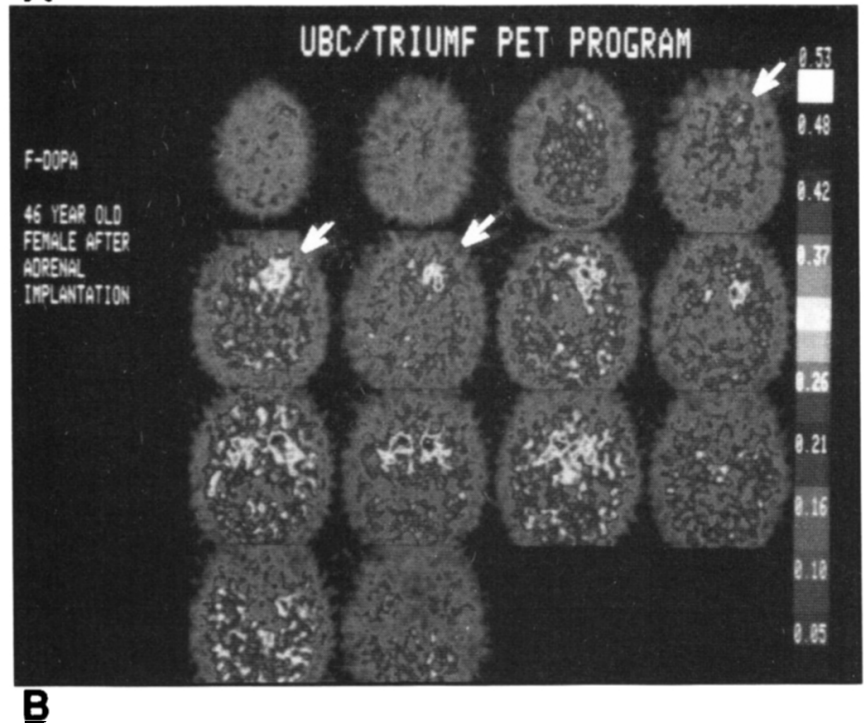

Figure 1 -6-FD PET scan of a 46-year-old female before $(A)$ and six weeks after $(B)$ adrenal implantation into the right caudate mucleus Note the accumulation of radioactivity in the frontal and striatal regions on the right post-operatively (arrows). Both scans show marked reduction of 6-FD accumulation consistent with parkinsonism. The orientation is that the left side of the scan is the anatomical left. The uppermost tomograph slice is on the top left and sequential images are of lower levels.

\begin{tabular}{|c|c|c|c|c|}
\hline Patient & Side & $\begin{array}{c}\text { Pre-operative 6-FD } \\
\text { Modified Ratio }\end{array}$ & $\begin{array}{c}\text { Post-operative 6-FD } \\
\text { Modified Ratio }\end{array}$ & Difference \\
\hline 1. & Right & 0.280 & 0.354 & $+26.4 \%$ \\
\hline \multirow[t]{2}{*}{2.} & Right & 0.509 & 0.439 & $-13.8 \%$ \\
\hline & Left & 0.413 & 0.297 & $-28.1 \%$ \\
\hline 3. & Right & 0.331 & 0.357 & $+7.6 \%$ \\
\hline 4. & Left & 0.321 & 0.306 & $-4.7 \%$ \\
\hline \multirow[t]{2}{*}{5.} & Right & 0.326 & 0.361 & $+11.0 \%$ \\
\hline & Left & 0.262 & 0.264 & $+1.0 \%$ \\
\hline
\end{tabular}

Difference not significant with Wilcoxon sign rank test.

Modified Ratio $=$ Radioactivity $($ Striatum - parietal - occipital cortex $)$ 


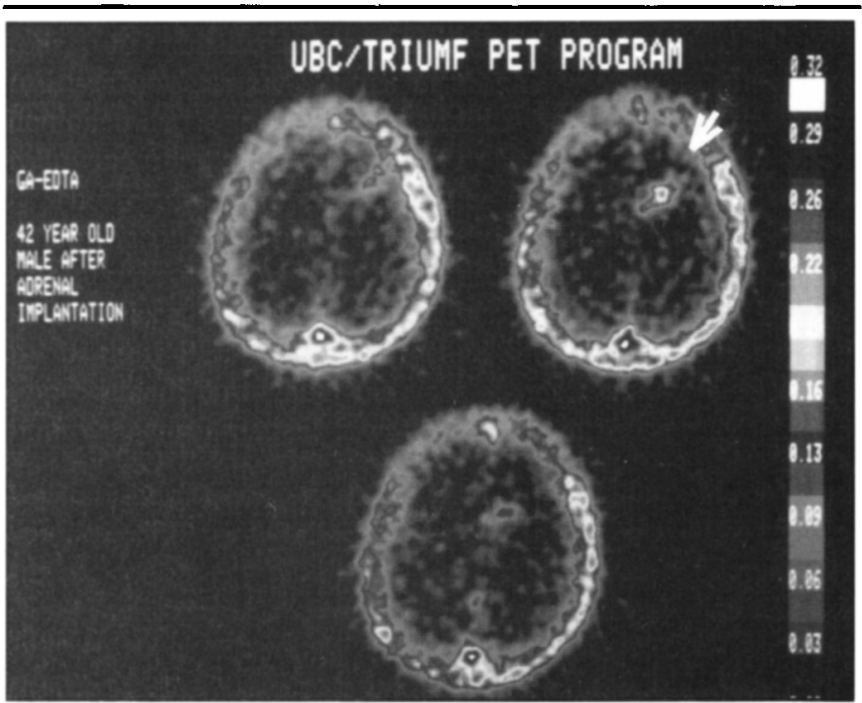

A

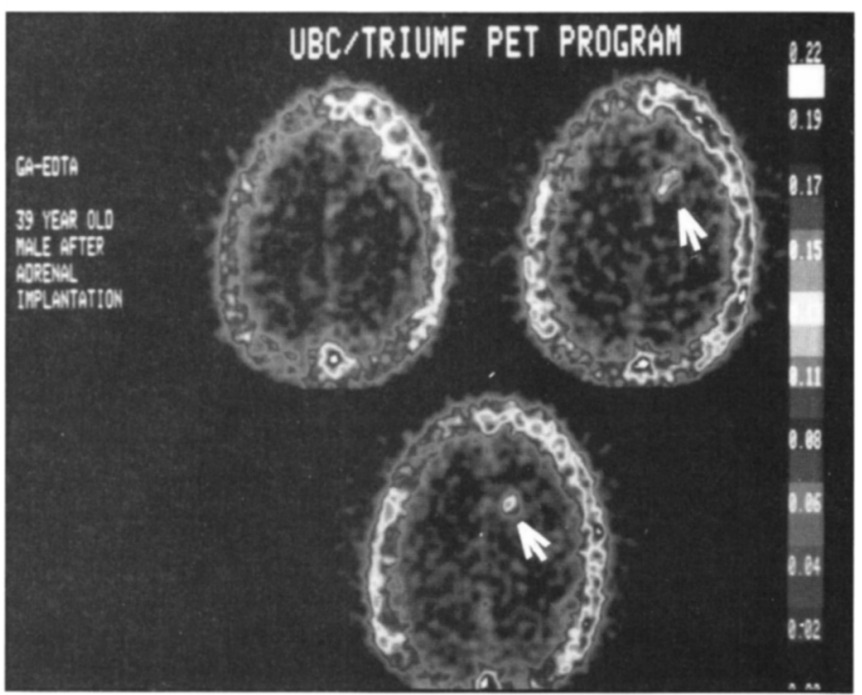

B

Figure 2 - Ga PET scans of a 42-year-old male (A) and a 39-year-old male $(B)$ after implantation surgery. The tomographic slices were chosen to represent the areas of the surgical tracts and implantation sites of the transplants. Note the accumulation of Ga-EDTA in these regions on the right (arrows).

the blood brain barrier through the same facilitated transport mechanism. Once past the blood brain barrier, 6-FD is taken up by dopaminergic neurons and converted to 6-fluorodopamine (FDA) by L-aromatic amino acid decarboxylase. Cumming and colleagues have shown that 6-FD and L-dopa have similar $\mathrm{K}_{\mathrm{m}}$ values for this enzyme. ${ }^{21}$ In regions without dopaminergic neurons, 6-FD remains as a component of the background radioactivity. Tomographic studies in monkeys ${ }^{22}$ and in man have demonstrated accumulation of radioactivity in the striatum 23 following 6-FD administration.

The results of the 6-FD studies suggest that a consistent alteration of the striatal radioactivity did not occur in these patients six weeks after adrenal medulla implantation. In the three subjects who demonstrated increases of striatal radioactivity in their PET studies only one showed signs of clinical improvement throughout the year of observation. The clinical improvement in patient 5 was not detected before the 3 month

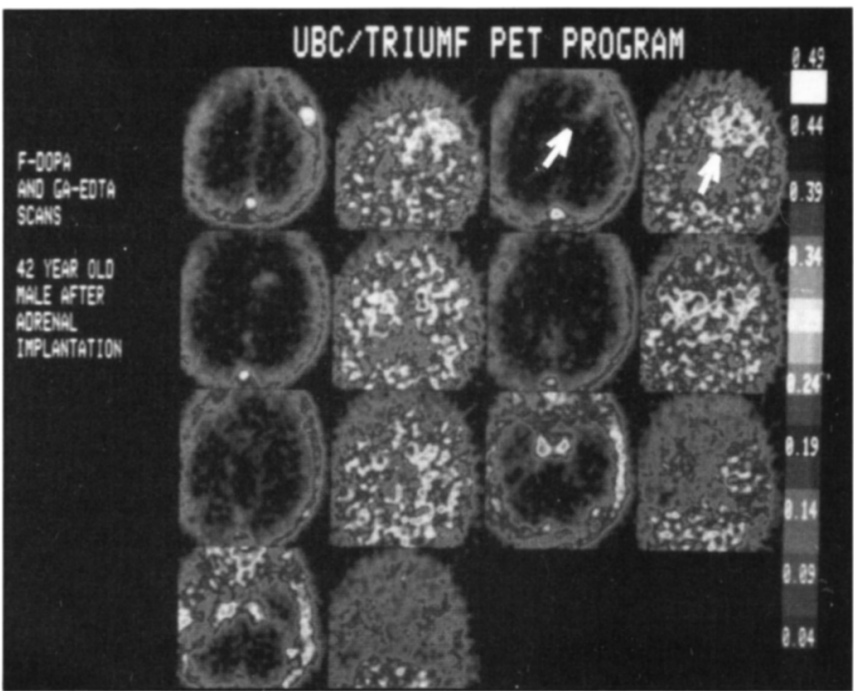

Figure 3 - 6-FD compared with Ga-EDTA PET scans on a 42-yearold male after surgery. The areas of Ga-EDTA permeability are the same as the areas of increased radioactivity after 6-FD administration (arrow's). This suggests abnormal 6-FD leakage across the blood-brain barrier at these sites post-operatively.

assessment. Her post-operative PET scan with 6-FD showed an increase on the side of the implant but this was smaller in magnitude than another subject who did not improve. The significance of this increase in radioactivity is difficult to interpret. 6FD PET studies therefore did not provide consistent correlation with the corresponding clinical examinations and did not provide an early indication of clinical improvement that would occur in the future.

It is possible that the striatal dopaminergic function was altered and was not appreciated by the current method of analysis. Small increases in dopaminergic funtion may occur that are within the error limitations of the semiquantitative analysis. A marginal increase in striatal dopamine content may potentially reverse the symptoms of Parkinson's disease in a patient who is close to the threshold for clinical symptomatology to occur.

Ga PET scans have been employed to evaluate the permeability of the blood brain barrier in two patients. The blood brain barrier normally has a low permeability to Ga. ${ }^{24}$ Experiments in rhesus monkeys with this tracer have demonstrated reversible breakdown of this barrier after unilateral carotid administration of hyperosmotic mannitol.24 Patients with cerebral infarction have been assessed with the same technique. ${ }^{25}$ It has been concluded that Ga PET scans were more sensitive to breakdown of the blood brain barrier than CT scans with contrast administration. These studies confirm the usefulness of this technique for the study of the regional integrity of the blood brain barrier using PET technology.

Animal models have been used to assess the permeability of the blood brain barrier after transplantation procedures. $26,27,28$ The cerebral vasculature has a continuous layer of endothelial cells connected by tight junctions. Adrenal medulla tissue and sympathetic ganglia do not have these junctions. Rosenstein has shown that the blood brain barrier does not form after transplantation of adrenal medulla 26 or autonomic ganglia up to nine months post-operatively. 27 These investigators have demonstrated that labelled dopamine could enter from the blood in the 
region of the graft. Furthermore, Rosenstein has reported that rat cerebral cortex grafted into fourth ventricle develops permanent barrier dysfunction regardless of the post-operative time studied. ${ }^{28}$ This observation suggests that even tissue with an initially competent blood brain barrier does not maintain this after implantation.

The present study indicates that breakdown of the blood brain barrier is present six weeks after surgery. It is not clear what influence this defect will have on the PET measurements. Increased permeability of the blood brain barrier may allow enhanced passage of 6-FD and 3-OMFD into the brain. This may be incorrectly interpreted as an increase in dopaminergic function at the site of implantation. Conversely, altered permeability may also permit passage of carbidopa into the affected regions, causing inhibition of 6-FD metabolism in that region.

It is possible that the increased regional permeability around the transplant tract and site may resolve at a later date. Longterm studies have not been performed in patients undergoing these procedures and the time course of the alteration is currently unknown. It is not clear if the defect seen on the Ga studies is from surgical alterations causing edema and gliosis or from the ultrastructural differences of the capillary endothelial network in the transplanted tissue.

In this study, the valid comparison of regional differences of radioactive accumulation after 6-FD administration (that may be interpreted as differences of dopaminergic function) are dependent on an intact blood brain interface in all regions. Using current methodology, interpretation of the presence or absence of changes of regional radioactivity uptake is difficult. It is possible that tracer kinetic analysis may provide further observations that will assist in the evaluation of dopaminergic function after neural implantation. This methodology, after suitable development and validation for 6-FD, may be able to independently assess transfer rates across the blood brain barrier and rates of metabolism of the radioligand in various regions. This will enable the differentiation of alterations of dopaminergic function from defects of the blood brain barrier. Unfortunately this analytic technique is not currently available.

\section{ACKNOWLEDGEMENTS}

We would like to thank Eileen Walsh, Poppy Schofield and Salma Jivan for their kind assistance in this project. This study was supported by the Medical Research Council of Canada (MRC) and the Dystonia Medical Research Foundation. Dr. Guttman was a Centennial Fellow of the MRC.

\section{REFERENCES}

1. Perlow MJ. Brain grafting as a treatment for Parkinson's disease. Neurosurg 1987; 20: 335-342.

2. Madrazo I, Drucker-Colin R, Diaz V, et al. Open microsurgical autograft of adrenal medulla to the right caudate nucleus in two patients with intractable Parkinson's disease. N Engl J Med 1987; 316: $831-834$

3. Olanow CW, Cahill D, Cox C. Autologous transplantation of adrenal medulla to caudate nucleus in Parkinson's disease. Neurology 1988; 38 (Suppl 1): 142.

4. Lieberman AN, Ransohoff J, Koslow M. Adrenal medullary to caudate nucleus transplant as an effective treatment for advanced Parkinson's disease (PD). Neurology 1988; 38 (Suppl 1): 142.

5. Goetz CG, Tanner CM, Penn RD, et al. Efficacy of intrastriatal adrenal medulla transplant in Parkinson's disease. Neurology 1988; 38 (Suppl 1): 142-143.

6. Watts RL, Bakay RAE, Iuvone PM, et al. Autologous adrenal- caudate transplantation in patients with Parkinson's disease (PD). Neurology 1988; 38 (Suppl 1): 143.

7. Allen GS, Burns RS, Tulipan NB, Parker RA. Adrenal medullary transplantation to caudate nucleus in Parkinson's disease: Initial clinical results in eighteen patients. Arch Neurol 1989 (in press).

8. Lindvall O, Backlund E-O, Farde L, et al. Transplantation in Parkinson's disease: Two cases of adrenal medullary grafts to the putamen. Ann Neurol 1987; 22: 457-468.

9. Tinter R, Clark K, Hom J, et al. Adrenomedullary transplantation in Parkinson's disease: Quantitative neurologic and biochemical investigation. Neurology 1988; 38 (Suppl 1): 143.

10. Carvey PM, Kroin JS, Zhang TJ, et al. Biochemical and immunochemical characterization of ventricular CSF from Parkinson's disease (PD) patients with adrenal medulla transplants in the patients. Neurology 1988; 38 (Suppl 1): 144.

11. Garnett ES, Firnau G, Chan PKH, et al. [18F]-fluorodopa, an analog of dopa, and its use in direct external measurements of storage, degeneration, and turnover of intracerebral dopamine. Proc Natl Acad Sci USA 1978; 75: 137-138.

12. Garnett ES, Nahmias C, Firnau G. Central dopaminergic pathways in hemiparkinsonism examined by Positron Emission Tomography. Can J Neurol Sci 1984; 11: 174-179.

13. Martin WRW, Stoess! AJ, Adam MF, et al. Positron Enission Tomography in Parkinson's Disease: Glucose and Dopa Metabolism. Adv Neurol 1986; 45: 95-98.

14. Leenders KL, Palmer AJ, Quinn N, et al. Brain dopamine metabolism in patients with Parkinson's disease measured with PET. J Neurol Neurosurg Psychiatry 1986; 49: 855-860.

15. Yahr MD, Duvoisin RC, Schear MJ, Barrett RE, Hoehn MM. Treatment of parkinsonism with levodopa. Arch Neurol 1969: 21: 343-354.

16. Evans B, Harrop R, Heywood D, et al. Engineering Developments on the UBC-TRIUMF Modified PETT VI Positron Emission Tomograph. IEEE Trans Nucl Sci 1982; NS-30: 707-710.

17. Adam MJ, Jivan S. Synthesis and purification of L-6-[18F] fluorodopa. J Appl Radiat Isotop (in press).

18. Firnau G, Sood S, Chirakal R, et al. Cerebral Metabolism of 6[18F] Fluoro-L-3, 4-Dihydroxyphenylalanine in the Primate. J Neurochem 1987; 48: 1077-1082.

19. Hardebo JE, Owman C. Barrier mechanisms for neurotransmitter monoamines and their precursors at the blood-brain interface. Ann Neurol 1980; 8: 1-11.

20. Martin WRW, Cumming P, Growchowski E, et al. Regional Inhomogeneity of Blood-Brain Barrier Transport Systems. J Cereb Blood Flow Metab 1987; 7 (Suppl 1): S332.

21. Cumming $P$, Hausser M, Martin WRW, et al. Kinetics of in virro decarboxylation and the in vivo metabolism of $2-18 \mathrm{~F}$ - and $6-18 \mathrm{~F}-$ fluorodopa in the hooded rat. Biochem Pharm 1988; 37: 247250.

22. Garnett ES, Firnau G, Nahmias $C$, et al. Striatal dopamine metabolism in living monkeys examined by positron emission tomography. Brain Res 1983; 280: 169-171.

23. Garnett ES, Firnau G, Nahmias C. Dopamine visualized in the basal ganglia of living man. Nature 1983; 305: 137-138.

24. Kessler RM, Goble JC, Bird JH, et al. Measurement of blood-brain barrier permeability with positron emission tomography and [68Ga] EDTA. J Cereb Blood Flow Metab 1984; 4: 3233-328.

25. Ericson K, Bergstrom M, Eriksson L, et al. Positron emission tomography with ${ }^{68} \mathrm{Ga}$-EDTA compared with transmission computed tomography in the evaluation of brain infarcts. Acta Radiologica 1981; 22: 385-398.

26. Rosenstein JM. Adrenal medulla grafts produce blood-brain barrier dysfunction. Brain Res 1987; 414: 192-196.

27. Rosenstein JM, Brightman MW. Alterations of the blood-brain barrier after transplantation of autonomic ganglia into the mammalian central nervous system. J Comp Neurol 1986; 250: 339351 .

28. Rosenstein JM. Neocortical transplants in the mammalian brain lack a blood-brain barrier to macromolecules. Science 1987 ; 235: $772-774$ 